Pacific Journal of Mathematic 


\title{
GENERALIZED AXISYMMETRIC ELLIPTIC FUNCTIONS
}

\author{
Peter A. McCoy
}

A generalized axisymmetric elliptic function (GASE) $\Psi_{\nu}: \Omega \subset E^{n} \rightarrow C$ of order $\nu \geqq 0$ solving the partial differential equation

$$
\mathscr{L}_{\nu}\left(\Psi_{\nu}\right) \equiv \frac{\partial^{2} \Psi_{\nu}}{\partial x^{2}}+\frac{\partial^{2} \Psi_{\nu}}{\partial \rho^{2}}+\frac{2 \nu}{\rho} \frac{\partial \Psi_{\nu}}{\partial \rho}+a(x) \frac{\partial \Psi_{\nu}}{\partial x}+c(x) \Psi_{\nu}=0
$$

with analytic coefficients is subject to Cauchy data: $\Psi_{\nu}(x, 0)=$ $f(x),(\partial / \partial \rho)\left(\Psi_{\nu}(x, 0)\right)=0$ along the singular line. These GASE may be generated from associated analytic functions of one complex variable or associated solutions to the corresponding nonsingular equation by certain integral operators. Convexity arguments geometrically characterize the values of GASE from those of the associates and kernel functions of the respective operators.

An extensive theory based on integral operators which characterizes the distribution of singularities of various classes of GASE from the distribution of singularities of their associates was developed by S. Bergman [2], R. P. Gilbert [3, 4], P. Henrici [6] and their colleagues [5]. Our aim is to apply convexity arguments from the analytic theory of polynomials of one complex variable to develop a geometric theory of the value distribution of GASE from the known value distribution of the associates. These results are based on two operators developed by Henrici [3, p. 199]; one which utilizes a kernel function to generate GASE from associated analytic functions of one complex variable and one which generates GASE of positive order from the associated GASE of order zero.

A theory connecting the values of axisymmetric harmonic polynomials (AHP) in $\boldsymbol{E}^{n}$ with those of associated polynomials of one complex variable was developed by M. Marden [8]. Gegenbauer's integral for ultraspherical polynomials was used to map polynomials of one complex variable onto AHP and then convexity arguments were used to relate their values. Using geometrical methods and R. P. Gilbert's operater $A_{\mu}$ [3, p. 168], the auther [10-12] utilized the conformal mapping properties of the associates to characterize sets of excluded values for generalized axisymmetric potentials (GASP) corresponding to solutions of (1) with $a(x) \equiv c(x) \equiv 0$.

Convexity arguments used in studying GASP were essentially independent of the kernel of the operator $A_{\mu}$ which is non-negative and dependent only on the variable of integration. In general, oper- 
ators transforming associated analytic functions of one complex variable into GASE have kernels which also depend on the circle in $\boldsymbol{E}^{n}$ on which the GASE is evaluated. By modifying previous convexity arguments, the influence of this additional dependence is geometrically characterized from the kernels and associates of the respective operators. Methods of refinement of certain types of bounds found in previous results [10-12] are also introduced.

1. Preliminaries. We shall be dealing with the cylindrical coordinates $(x, \rho, \phi)$,

$$
x=x_{1}, \quad \rho^{2}=x_{2}^{2}+\cdots+x_{n}^{2}, \quad 0 \leqq \phi \leqq 2 \pi
$$

and spherical coordinates $(r, \phi, \theta)$ where

$$
x=r \cos \theta, \quad \rho=r \sin \theta .
$$

We shall be considering analytic functions $f$ of one complex variable whose natural domains are taken as open simply connected axiconvex sets $\omega \subset C$. That is, $\zeta \in \omega$ if and only if $\zeta t+\bar{\zeta}(1-t) \in \omega$, $0 \leqq t \leqq 1$, and GASE $\Psi_{\text {, }}$ whose natural domains are axisymmetric sets $\Omega \subset E^{n}$ generated by rotating axiconvex sets about the $x$-axis.

As is well known, Henrici [6, p. 21] has shown that a family of GASE $\left\{\Psi_{\nu}\right\}_{\nu \geq 0}$ on $\Omega$ may be generated from the associate $\Psi_{0}$ via the operator

$$
\Psi_{\nu}(x, \rho)=\alpha_{\nu} \int_{0}^{\pi} \Psi_{0}(x, \rho \cos t)(\sin t)^{2 \nu-1} d t
$$

with normalization

$$
\alpha_{\nu}^{-1}=\int_{0}^{\pi}(\sin t)^{2 \nu-1} d t=\sqrt{\pi} \Gamma(\nu) / \Gamma(\nu+1 / 2)
$$

where $\Psi_{\nu}$ is extended as an even function in $\rho$.

In our study it will be convenient to refer to the circles

$$
X=\{(x, \rho, \phi) \mid 0 \leqq \phi \leqq 2 \pi\}
$$

and

$$
X_{*}=\left\{\left(x_{*}, \rho_{*}, \phi\right) \mid 0 \leqq \phi \leqq 2 \pi\right\}
$$

where

$$
X \prec X_{*} \Longleftarrow x=x_{*} \text { and } \rho \leqq \rho_{*} \cdot
$$

Whenever $X_{*}$ is in $\Omega, \Psi_{0}\left(\left\{X \mid X \prec X_{*}\right\}\right)$ is a curve in $C$ and $\mathscr{C}\left\{\Psi_{0}\left(X_{*}\right)\right\}$ 
is its closed convex hull. A refinement of $\left.\Gamma=P_{\mathcal{B}}\left\{\Psi_{*}\right)\right\}$ is provided by

$$
\mathscr{S}\left\{\Psi_{0}\left(X_{*}\right)\right\}=\Gamma-\partial_{0}[\Gamma]
$$

where

$$
\hat{o}_{0}[\Gamma]=\left\{\zeta \in \partial \Gamma \mid \exists \xi, \eta \in \partial \Gamma \ni \zeta=\xi t+(1-i) r_{\gamma}, 0<t<1\right\}
$$

is the set of extreme points on the boundary of $\Gamma$. We now turn to

2. Distribution of values of GASE.

THEOREM 1. Let $\left\{\Psi_{2}\right\}_{2 \geqq 0}$ be a family of GASE with domain $\Omega$. Then for each circle $X_{*}$ in $\Omega$

$$
\Psi_{\nu}(X) \in \mathscr{S}\left\{\Psi_{0}\left(X_{*}\right)\right\}
$$

for all circles $X \prec X_{*}$ and for all orders $\nu \geqq 0$. In particular, the only possible zeros of $\Psi_{\nu}$ occur on those circles $X_{*}$ for which $\mathscr{S}\left\{\Psi_{0}\left(X_{*}\right)\right.$ contains the origin.

Proof. Transposing terms in (2) leads to

$$
\int_{0}^{\pi}\left[\Psi_{0}\left(x_{*}, \rho_{*} \cos t\right)-\Psi_{\nu}(X)\right](\sin t)^{2 \nu-1} d t=0 \quad X \prec X_{*} .
$$

Let us assume that for some order $\nu>0, \Psi_{\nu}(X) \in \mathscr{S}\left\{\Psi_{0}\left(X_{*}\right)\right\}$. Under these conditions, the following inequality is satisfied for $t \in(0, \pi) \equiv I$,

$$
\arg \left[\Psi_{0}\left(x_{*}, \rho_{*} \cos t\right)-\Psi_{\nu}(X)\right]<\pi .
$$

In addition, the integrand of (2) contains $(\sin t)^{2 \nu-1}$, a positive factor for $t \in I$, which when combined with (3) produces

$$
\arg \left\{\left[\Psi_{0}\left(x_{*}, \rho_{*} \cos t\right)-\Psi_{\nu}(X)\right](\sin t)^{2 \nu-1}\right\}<\pi
$$

for $t \in I$. In view of this inequality, we may follow the reasoning found in [9] to conclude that the integrand of (2) considered as the limit of a sum of vectors which terminate in a convex sector with vertex at $\Psi_{\nu}(X)$, cannot vanish, a contradiction to (2). Observing that the inclusion $\mathscr{S}\left\{\Psi_{0}(X)\right\} \subset \mathscr{S}\left\{\Psi_{0}\left(X_{*}\right)\right\}$ holds for all circles $X \prec X_{*}$ completes the proof.

As consequences of Theorem 1, let us now derive the following corollaries.

COROLLARY 1.1. For each $\alpha,\left\{\Psi_{\nu}^{\alpha}\right\}_{\nu \geqq 0}$ is a family of GASE with domain $\Omega$. If the associates of these families assume values on $\Omega$ which lie in mutually exclusive closed convex sets, then for all circles $X$ and 
$X^{\prime}$ in $\Omega$ and all orders $\mu$ and $\nu$,

$$
\Psi_{\mu}^{\alpha}(X) \neq \Psi_{\nu}^{\beta}\left(X^{\prime}\right)
$$

for all distinct $\alpha$ and $\beta$.

Proof. This follows from the fact that for $X$ in $\Omega$

$$
\mathscr{S}\left\{\Psi_{\gamma}(X)\right\} \subset \mathscr{C}\left\{\Psi_{\gamma}(X)\right\}, \quad \gamma=\alpha, \beta,
$$

and that from Theorem 1

$$
\mathscr{C}\left\{\Psi_{0}^{\alpha}(X)\right\} \cap \mathscr{C}\left\{\Psi_{0}^{\beta}\left(X^{\prime}\right)\right\}=\varnothing
$$

when $\alpha$ and $\beta$ are distinct.

In the special case in which the associate of a family of GASE reduces to a finite linear combination of GASE of order zero, a result analogous to those of Marden and Walsh [7, p. 74] which consider the null sets of linear combinations of polynomials of one complex variable is found in

COROLLARY 1.2. If the associate of a family of GASE $\left\{\Psi_{\nu}\right\}_{\nu \geq 0}$ with domain $\Omega$ is represented as $\Psi_{0}=\sum_{i=1}^{n} a_{i} \Psi_{0}^{i}$ where $\Psi_{0}^{i}$ are GASE on $\Omega$ for $1 \leqq i \leqq n$, then

$$
\Psi_{\nu}(X)=\sum_{i=1}^{n} a_{i} \Psi_{\nu}^{i}(X), \quad X \in \Omega
$$

where $\Psi_{\nu}^{i}(X) \in \mathscr{C}\left\{\Psi_{0}^{i}\left(\Omega_{0}\right), 1 \leqq i \leqq n\right.$, for all orders $\nu$ and circles $X$ in compact axiconvex subsets $\Omega_{0}$ of $\Omega$.

Proof. We identify each GASE $\Psi_{\nu}^{i}$ with its associate $\Psi_{0}^{i}$ and

$$
\mathscr{C}\left\{\sum_{i=1}^{n} a_{i} \Psi_{0}^{i}\left(\Omega_{0}\right)\right\}=\sum_{i=1}^{n} a_{1} \mathscr{C}\left\{\Psi_{0}^{i}\left(\Omega_{0}\right)\right\}
$$

These results may be recast to provide an analytical description of the $\gamma$-circles of a GASE, that is, of circles $X$ on which $\Psi_{\nu}$ assumes the value $\gamma$. Let us consider

CoROLlaRY 1.3. Let the family of GASE $\left\{\Psi_{\nu}\right\}_{\nu \geqq 0}$ be defined on $\Omega$. Then the only possible $\gamma$-circles of $\Psi_{\nu}$ on $\Omega$ are circles $X_{*}$ which are $\gamma$-circles of $\Psi_{0}$ or which include circles $X \prec X_{*}$ on the locus

$$
\arg \left(\frac{\Psi_{0}\left(X_{*}\right)-\gamma}{\Psi_{0}(X)-\gamma}\right)= \pm \pi
$$

Proof. If there exists a circle $X_{*}$ in $\Omega$ for which $\Psi_{0}\left(X_{*}\right)=\gamma$, then $\gamma$ is in $\mathscr{C}\left\{\Psi_{0}\left(X_{*}\right)\right\}$ and consequently $X_{*}$ is a possible zero of 
$\Psi_{\nu}-\gamma$. On the other hand, if there exists a circle $X \prec X_{*}$ on the locus

$$
\arg \left(\Psi_{0}\left(X_{*}\right)-\gamma\right)= \pm \pi+\arg \left(\Psi_{0}(X)-\gamma\right),
$$

then the image of the disk $\left\{X \mid X \prec X_{*}\right\}$ under $\Psi_{0}-\gamma$ is a curve whose closed convex hull includes the origin. As this exhausts the cases for which $\mathscr{C}\left\{\Psi_{0}(X)\right\}$ meets the origin, the only possible zeros of $\Psi_{\nu}-\gamma$ satisfy the above locus.

We next establish that locally the distribution of values of GASE can be made independent of the associate.

COROLLARY 1.4. Let $\left\{\Psi_{\mu}^{\alpha}\right\}_{\mu \geq 0}$ and $\left\{\Psi_{\nu}^{\beta}\right\}_{\nu \geq 0}$ be families of GASE which are generated from distinct associates on $\Omega$. If for some orders $\mu$ and $\nu$, the values assumed by $\Psi_{\mu}^{\alpha}$ and by $\Psi_{\nu}^{\beta}$ on a segment $J$ of the axis of symmetry are separated by a line, there exists an axiconvex set $\Omega_{J} \subset \Omega$ on which

$$
\Psi_{\mu}^{\sim}\left(\Omega_{J}\right) \cap \Psi_{\nu}^{\beta}\left(\Omega_{J}\right)=\varnothing
$$

for all orders $\mu$ and $\nu$.

Proof. From (2) we find that $\Psi_{\mu}^{\alpha}(X)=\Psi_{0}^{\alpha}(X)$ and $\Psi_{\nu}^{\beta}(X)=\Psi_{0}^{\beta}(X)$ for all positive orders whenever $X$ is a point of $J$. Since a line $\mathscr{L}$ separates the curves $\Psi_{\mu}^{\alpha}(J)$ and $\Psi_{\nu}^{\beta}(J)$, there are axiconvex sets $\Omega^{\alpha}$ and $\Omega^{\beta}$ in $\Omega$ for which $\mathscr{S}\left\{\Psi_{0}^{\alpha}(X)\right\} \cap \mathscr{S}\left\{\Psi_{0}^{\beta}\left(X^{\prime}\right)\right\}=\varnothing$ for all circles $X$ in $\Omega^{\alpha}$ and $X^{\prime}$ in $\Omega^{\beta}$. Defining $\Omega_{J}=\Omega^{\alpha} \cap \Omega^{\beta}$ completes the proof.

Results having more immediate application can be deduced from a second operator introduced by Henrici [3, p. 201] which generates a family $\left\{F_{\nu}\right\}_{\nu \geq 0}$ of GASE from an associated analytic function $f$ and the Riemann function $k_{\nu}$ for (1). This operator takes the form

$$
F_{\nu}(X)=c_{\nu} \int_{0}^{\pi} k_{\nu}(X, t) f(\sigma) d t
$$

where $c_{\nu}$ is a normalizing constant and $\sigma=x+i \rho \cos t, 0 \leqq t \leqq 2 \pi$.

An extension of the previous method allows a characterization of the values of $F_{\nu}$ in terms of the product of the integral of the Riemann function and a function depending on $f$. The advantage of this approach lies in the fact that since the integral of $k_{\nu}$ is independent of $f$, the qualitative properties of $F_{\nu}$ may be determined from the conformal mapping properties of $f$.

To accomplish this, let us restrict our attention to axisymmetric sets $\Omega_{\nu} \subset \Omega$ on which the Riemann function satisfies 


$$
\arg \left\{k_{\nu}(X, t)\right\} \leqq \pi-\gamma_{\nu}
$$

for $X \in \Omega_{\nu}$ and $t \in I$. Whenever (7) holds and the associate $f$ is analytic on the corresponding $\omega_{\nu} \subset C$, the family $\left\{F_{\nu}\right\}_{\nu \geqq 0}$ is said to be $\gamma_{\nu}$-convex on $\Omega_{\nu}$. The angle $\gamma_{\nu}$ and the set $\Omega_{\nu}$ may be independent of the order $\nu$ as is the case for GASP where $k_{\nu}(X, t)$ reduces to $(\sin t)^{2 \nu-1}$ :

The point set for which $f\left(\omega_{\nu}\right)$ subtends an angle of at least $\gamma_{\nu}$ is designated by

$$
\mathscr{S}\left[f\left(\omega_{\nu}\right), \gamma_{\nu}\right]
$$

We now turn to

THEOREM 2. Let $\left\{F_{\nu}\right\}_{\nu \geqq 0}$ be a family of GASE which is $\gamma_{2}$-convex on $\Omega_{\nu}$. Then on $\Omega_{\nu}$, each $F_{\nu}$ may be represented as

$$
F_{\nu}(X)=c_{\nu} \eta(X) \int_{0}^{\pi} k_{\nu}(X, t) d t
$$

where $\eta(X) \in \mathscr{S}\left[f\left(\omega_{\nu}\right), \gamma_{\nu}\right]$. If $\mathscr{S}\left[f\left(\omega_{\nu}\right), \gamma_{\nu}\right]$ does not meet the origin, $F_{\nu}$ has no zeros on $\Omega_{\nu}$.

Proof. For each circle $X$ in $\Omega_{\nu},(7)$ holds. Hence, $\int_{0}^{\pi} k_{\nu}(X, t) d t \neq 0$ which permits the function $\eta$ to be defined by (8). As in Theorem 1, a contradiction is reached if (8) is rewritten as

$$
\int_{0}^{\pi} k_{\nu}(X, t)[f(\sigma)-\eta(X)] d t=0
$$

and we assume that $\eta(X) \notin \mathscr{S}\left[f\left(\omega_{\nu}\right), \gamma_{\nu}\right]$ since then

$$
\arg \left\{k_{\nu}(X, t)[f(\sigma)-\eta(X)]\right\}<\pi
$$

for $t \in I$.

Null circles of GASE with polynomial associates are connected with the zeros of their associates through

COROLlary 2.1. Let $\left\{F_{\nu}\right\}_{\nu \geqq 0}$ be a family of GASE which is $\gamma_{\nu}$ convex on $\Omega_{\nu}$ have $f$, a polynomial of degree $n$, as associate. If the circle $X$ is a zero of $F_{\nu}, f$ has at least one zero in the circle

$$
|\zeta-x| \leqq \rho \cot \left(\gamma_{\nu} / n\right), \quad \zeta \in C \text {. }
$$

Proof. Let us factor the integrand of (9) as

$$
f(\sigma)-\eta(X)=\alpha_{0} \prod_{j=1}^{n}\left(\sigma-\alpha_{j}\right)
$$

Assuming that $\left|\alpha_{j}-x\right|>\rho \cot \left(\gamma_{\nu} / n\right), 1 \leqq j \leqq n$, leads us to the inequality 


$$
\arg \left\{\alpha_{0}^{-1}(f(\sigma)-\eta(X))\right\}<\left(\gamma_{\nu} / n\right) n
$$

for $t \in I$. Since $k_{\nu}$ is $\gamma_{\nu}$-convex on $\Omega_{\nu}$, we arrive at (14) which leads to a contradiction of (12) by the usual argument.

We next find that a partial analog of the Riemann mapping theorem is included in

CoROLlaRy 2.2. Let $\Omega$ be an axisymmetric subset of $\boldsymbol{E}^{n}$ with axis of symmetry $J$. Then for any point $X_{0}$ on $J$, there is a GASE $F_{n}$ with domain $\Omega$ such that $F_{n}(J)$ is a simple curve thru the origin, $(\partial / \partial x)\left(F_{n}\left(X_{0}\right)\right)>0$ and

$$
\left|F_{n}(X)\right|<1
$$

for all $X$ in $\Omega$.

Proof. Let $\omega \subset C$ be the axiconvex set associated with $\Omega$ and $I$ be its axis of symmetry. The Riemann mapping theorem assets the existence of a one-one analytic function $f$ with domain $\omega$ such that if $\left(x_{0}, 0\right) \in I, f\left(x_{0}, 0\right)=0, f_{x}\left(x_{0}, 0\right)>0$ and $|f(\zeta)|<1$ for all $\zeta$ in $\omega$.

GASP are generated by $(10)$ from $k_{n}(X, t)=(\sin t)^{2 n-1}$. Since the values attained by $F_{n}$ on $J$ and $f$ on $I$ agree and $F_{n}$ has no singularities on $\Omega$ [3, p. 179], we establish from (12) that for $X$ in $\Omega$,

$$
\begin{aligned}
\left|F_{n}(X)\right| & \leqq \alpha_{n}^{-1} \int_{0}^{\pi}|f(\sigma)|(\sin t)^{2 n-1} d t \\
& <\alpha_{n}^{-1} \int_{0}^{\pi}(\sin t)^{2 n-1} d t=1
\end{aligned}
$$

These results are applied to

3. Generalized axisymmetric Helmholtz functions. The generalized axisymmetric Helmholtz functions (GASH) arise as solutions to

$$
\frac{\partial^{2} u}{\partial x^{2}}+\frac{\partial^{2} u}{\partial \rho^{2}}+\frac{2 \nu}{\rho} \frac{\partial u}{\partial \rho}+k^{2} u=0, k, \nu>0 .
$$

It is well known $[6$, p. 26] that GASH are generated thru the operator

$$
F_{\nu}(X)=c_{\nu} \int_{0}^{\pi} J_{\nu-1}(k \rho \sin t) f(k \sigma)(\sin t)^{\nu} d t
$$

where

$$
c_{\nu}=(k \rho / 2)^{1-\nu} \Gamma(\nu+1 / 2) / \Gamma(1 / 2) .
$$

This operator establishes a correspondence between the Neumann 
series expansion of analytic functions $f$ regular about the origin [3, p. 214]

$$
f(k \sigma)=(k \sigma)^{-\nu} \sum_{n=0}^{\infty} a_{n} J_{\nu+n}(k \sigma)
$$

and the GASH represented by the Bessel-Gegenbauer series

$$
F_{\nu}(x, \rho) \equiv \widetilde{F}_{\nu}(r, \theta)=d_{\nu} \sum_{n=0}^{\infty} \frac{a_{n} n !}{\Gamma(2 \nu+n)} J_{\nu+n}(k r) C_{n}^{\nu}(\cos \theta)
$$

where $d_{\nu}=\Gamma(2 \nu)(k r)^{-\nu}$.

If $\xi_{\nu-1}$ denotes the smallest positive zero of the Bessel function $J_{\nu-1}$ of order $\nu-1, \arg \left\{J_{\nu-1}(\lambda)\right\}$ is constant for $0<\lambda<\xi_{\nu-1}$. Hence, Theorem 2 permits us to deduce.

THEOREM 3. Let $\left\{F_{\nu}\right\}_{\nu \geqq 0}$ be a family of GASE with domain $\Omega$ and associate $f$. Then on all circles common to $\Omega$ and the cylinder $\rho \leqq \xi_{\nu-1} k^{-1}, F_{\nu}$ may be represented as

$$
F_{\nu}(x, \rho)=2^{\mu} \Gamma(\mu+1) \eta(x, \rho) \frac{J_{\mu}(k \rho)}{(k \rho)^{\mu}}
$$

where $\mu=\nu-1 / 2$ and $\eta(x, \rho) \in \mathscr{C}\{f(k \omega)\}$. If $\mathscr{C}\{f(k \omega)\}$ does not contain the origin, $F_{\nu}$ has no zeros on $\Omega$ for $\rho \leqq \xi_{\nu-1} k^{-1}$.

Proof. Sonine's first integral allows the evaluation

$$
\int_{0}^{\pi} J_{\nu-1}(k \rho \sin t)(\sin t)^{\nu} d t=\Gamma(1 / 2)(2 / k \rho)^{1 / 2} J_{\nu-1 \mid 2}(k \rho) .
$$

By (12), we are permitted to define the function $\eta$ in the cylinder $\rho \leqq \xi_{\nu-1} k^{-1}$ by

$$
\lambda_{\nu} \int_{0}^{\pi} f(k \sigma) J_{\nu-1}(k \rho \sin t)(\sin t)^{\nu} d t=c_{\nu} \eta(x, \rho) J_{\nu-1}(k \rho) /(k \rho)^{\nu-1 \mid 2}
$$

where

$$
\lambda_{\nu}=(k \rho / 2)^{1-\nu} \Gamma(\nu+1 / 2) / \Gamma(1 / 2)
$$

since

$$
\arg \left\{J_{\nu-1}(k \rho \sin t)(\sin t)^{\nu}\right\}=0, \quad t \in I
$$

implies that (21) is nonvanishing. By rearranging terms in (22), we find that if $c_{\nu}=2^{\nu-1 / 2} \Gamma(\nu+1 / 2)$, (21) becomes

$$
\int_{0}^{\pi}[f(k \sigma)-\eta(x, \rho)] J_{\nu-1}(k \rho \sin t)(\sin t)^{\nu} d t=0 .
$$


The previous argument applies and the proof is complete.

As a further application of this method, let us consider the following

ExAmple. For $\nu>0$, let the GASH $F_{\nu}$ be defined on $E^{n}$ by

$$
F_{\nu}(X)=e_{\nu} \sum_{n=0}^{\infty} \frac{(\nu+n) n ! i^{n}}{\Gamma(2 \nu+n)} C_{n}^{\nu}(k) C_{n}^{\nu}(\cos \theta) J_{\nu+n}(k \rho)
$$

where

$$
e_{\nu}=2^{\nu} \Gamma(\nu) \Gamma(2 \nu) r^{-\nu} \text {. }
$$

By (18), the associate of $F_{\nu}$ has the expansion

$$
f(i k \sigma)=2^{\nu} \Gamma(\nu)(k \sigma)^{-\nu} \sum_{n=0}^{\infty} i^{n}(\nu+n) C_{n}^{\nu}(k) J_{\nu+n}(k \sigma)
$$

which by $[1$, p. 64] we identify as the Neumann series $f(i k \sigma)=$ $\exp (i k \sigma)$. Since $f(i k \sigma)=f\left(i k\left(\sigma+2 \pi j k^{-1}\right)\right)$ for $j=0, \pm 1, \pm 2, \cdots$, the image of the union $\omega(k)$ of the rectangles

$$
\omega_{j}(k)=\left\{\xi+i \eta|| \eta\left|<\xi_{\nu-1} k^{-1},\right| \xi^{*} \mid<\pi k^{-1}, \xi=\xi^{*}+2 \pi j k^{-1}\right\}
$$

under $f$ lies in the annular region

$$
\mathscr{A}(k)=\left\{\zeta|| \arg \zeta\left|<\pi k^{-1}, \exp \left(-\xi_{\nu-1} k^{-1}\right)<\right| \zeta \mid<\exp \left(\xi_{\nu-1} k^{-1}\right)\right\}
$$

provided $k>1$. In the event that $k \leqq 1, \omega(k)$ reduces to the infinite strip $|\eta|<\xi_{\nu-1} k^{-1}$ and $\mathscr{A}(k)$ the annulus $\exp \left(-\xi_{\nu-1} k^{-1}\right)<|\zeta|<$ $\exp \left(\xi_{\nu-1} k^{-1}\right)$. It is easily seen from (18) and the periodicity of $f$ that

$$
F_{\nu}(x, \rho)=F_{\nu}\left(x+2 \pi j k^{-1}, \rho\right), \quad j=0, \pm 1, \pm 2, \cdots .
$$

Theorem 3 permits us to conclude that on $\Omega(k)$, the union of the truncated cylinders $\Omega_{j}(k)$ generated by rotating $\omega_{j}(k)$ into $\boldsymbol{E}^{n}$, the GASH (24) reduces to

$$
F_{\nu}(x, \rho)=2^{\mu} \Gamma(\mu+1) \eta(x, \rho) J_{\mu}(k \rho) /(k \rho)^{\mu}
$$

where $\eta$ assumes its values in $\mathscr{A}(k)$.

A natural question arising from Corollary 2.1 is that of recovering the distribution of values of the associate from that of the GASH. Such a connection is revealed thru the inverse operator [3, p. 216]

$$
f(k \sigma)=l_{\nu} \int_{-1}^{+1} k^{\prime}(\sigma, r, \xi) F_{\nu}\left(r \xi, r\left(1-\xi^{2}\right)^{1 / 2}\right) d \xi
$$

with $l_{\nu}=\Gamma(2 \nu)(k r)^{-1}$ where the kernel $k^{\prime}$ is modified so as to avoid 
singularities. The GASH $F_{\nu}$ and consequently $f$ are entire functions. Since $\arg (d \xi)$ is constant for $\xi \in(1,1)$ it follows that

THeorem 4. Let $f$ and $F_{\text {, }}$ be entire functions. If for $\sigma \in \boldsymbol{C}$, $r \in(0, R]$ and $\xi \in(-1,+1), \arg \left\{k^{\prime}(\sigma, r, \xi)\right\} \leqq \pi-\gamma$, then $f$ may be represented by

$$
f(k \sigma)=l_{\nu} \eta(r) \int_{-1}^{+1} k(\sigma, r, \xi) d \xi
$$

where $\eta(r) \in \mathscr{S}\left[\bigcup_{|\xi| \leqq 1} F_{\nu}(r \xi, r|\xi|), \gamma\right]$.

4. Analytic continuation of solutions. Gilbert [4] discusses methods of analytic continuation of his operators by allowing the coordinates $(x, \rho, \phi)$ to attain complex values thru deformation of the contour of integration so as to avoid singularities of the integrand. The methods used here may be adopted to that approach provided that the deformed contour $\mathscr{L}_{*}$ is sufficiently smooth so that the variation if $\arg (d \xi)$ over $\mathscr{L}_{*}, \mathscr{V}\{\arg (d \xi)\}_{\mathscr{L}_{*}}$, is small or there are factors in the integrand which compensate for $\mathscr{V}\{\arg (d \xi)\}_{\mathscr{E} *}$.

This situation arises in the case of GASP $u_{\nu}$ on $\Omega$ generated by Gilbert's operator [3, p. 168]

$$
u_{\nu}(x, \rho)=\alpha_{\nu}^{-1} \int_{\mathscr{L}} f(\tau)\left(\zeta-\zeta^{-1}\right)^{2 \nu-1} \zeta^{-1} d \zeta
$$

with $\tau=x+(i \rho / 2)\left(\zeta-\zeta^{-1}\right)$ and $\mathscr{L}=\{\zeta=\exp (i \theta) \mid 0 \leqq \theta \leqq \pi\}$. On $\mathscr{L}, \mathscr{V}\left\{\arg \left(\zeta^{-1} d \zeta\right\}_{\mathscr{L}}\right.$ and $\mathscr{V}\left\{\arg \left(\zeta-\zeta^{-1}\right)\right\}_{\mathscr{L}}$ are constant. If $u_{\nu}$ is continued beyond $\Omega$ by fixing the endpoints of $\mathscr{L}$ and continuously deformed into $\mathscr{L}_{*}$, an ellipse with eccentricity $\varepsilon \ll 1$, continuity guarantees that $\mathscr{V}\left\{\arg \left(\zeta^{-1} d \zeta\right)\right\}_{\mathscr{L}_{*}}$ and $\mathscr{V}\left\{\arg \left(\zeta-\zeta^{-1}\right)\right\}_{\mathscr{L}_{*}}$ are small so that previous reasoning can be applied to obtain a result analogous to Theorem 2 on the continued domain $\Omega_{*}$. See [11].

\section{REFERENCES}

1. H. Bateman, Higher Transcendental Functions, Bateman Manuscript project, ed. A. Erdelyi, McGraw-Hill, New York, 1953.

2. S. Bergman, Integral Operators in the Theory of Linear Partial Differential Equations, Ergebnisse der Mathematik and ihrer Grenzebrite, N. F., Heft 23, SpringVerlag, Berlin, 1961.

3. R.P. Gilbert, Function Theoretic Methods in the Theory of Partial Differential Equations, Mathematics In Science and Engineering, vol. 54, Academic Press, New York, 1969.

4. - Constructive Methods in Elliptic Equations, Lecture Notes in Mathematics \#365, Springer-Verlag, New York, 1974.

5. R. P. Gilbert and R. Newton, Analytic Methods in Mathematical Physics, Gordon 
and Breach Science Publishes, New York, 1970.

6. P. Henrici, Elliptic Equations with Singular Coefficients, Boundary Value Problems in Differential Equations, ed. R. E. Langer, Univ. of Wisconsin Press, Madison, Wisc. 1960.

7. Morris Marden, Geometry of polynomials, Math. Surveys, no. 3, Amer. Math. Soc., Providence, R. I., 1966.

8. - Value distributions of harmonic Polynomials in several real variables, Trans. Amer. Math. Soc., 159, Sept. 1971.

9. ——, A generalization of Weierstrass' and Fekete's mean value theorems, Bull Amer. Math. Soc., 38, (1932).

10. P.A. McCoy, Value distribution of axisymmetric potentials, Amer. J. Math., XCV, Summer 1973.

11. Value distribution of potentials in three real variables, Proc. Amer. Math. Soc., 36

12. - Value distribution of generalized axisymmetric potentials, to appear, J. Approx. Theory.

Received February 22, 1974 and in revised form March 7, 1975. This paper was supported by the Naval Academy Research Council. Presented to the American Mathematical Society, January 17, 1974.

U. S. Naval ACademy, Annapolis, maryland 



\section{PACIFIC JOURNAL OF MATHEMATICS}

\section{EDITORS}

RICHARD ARENS (Managing Editor) University of California

Los Angeles, California 90024

\section{R. A. Beaumont}

University of Washington Seattle, Washington 98105
J. DugundJI Department of Mathematics University of Southern Californıa Los Angeles, California 90007

D. Gilbarg aNd J. Milgram Stanford University Stanford, California 94305

\section{ASSOCIATE EDITORS}

E. F. BeCKENBACH

B. H. NeUmanN

F. Wolf

K. YoSHIDA

\section{SUPPORTING INSTITUTIONS}

UNIVERSITY OF BRITISH COLUMBIA CALIFORNIA INSTITUTE OF TECHNOLOGY UNIVERSITY OF CALIFORNIA MONTANA STATE UNIVERSITY UNIVERSITY OF NEVADA NEW MEXICO STATE UNIVERSITY OREGON STATE UNIVERSITY UNIVERSITY OF OREGON OSAKA UNIVERSITY
UNIVERSITY OF SOUTHERN CALIFORNIA STANFORD UNIVERSITY UNIVERSITY OF TOKYO UNIVERSITY OF UTAH WASHINGTON STATE UNIVERSITY UNIVERSITY OF WASHINGTON AMERICAN MATHEMATICAL SOCIETY NAVAL WEAPONS CENTER 


\section{Pacific Journal of Mathematics \\ Vol. 59, No. 1 \\ May, 1975}

Shashi Prabha Arya and M. K. Singal, More sum theorems for topological

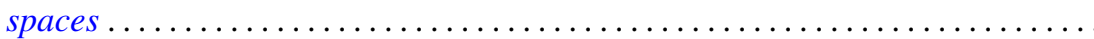

Goro Azumaya, F. Mbuntum and Kalathoor Varadarajan, On M-projective and

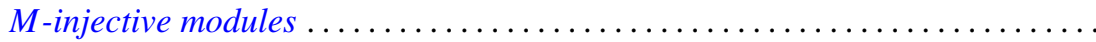

Kong Ming Chong, Spectral inequalities involving the infima and suprema of

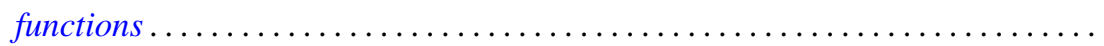

Alan Hetherington Durfee, The characteristic polynomial of the monodromy .......

Emilio Gagliardo and Clifford Alfons Kottman, Fixed points for orientation

preserving homeomorphisms of the plane which interchange two points ......

Raymond F. Gittings, Finite-to-one open maps of generalized metric spaces .......

Andrew M. W. Glass, W. Charles (Wilbur) Holland Jr. and Stephen H. McCleary,

$a^{*}$-closures of completely distributive lattice-ordered groups .............

Matthew Gould, Endomorphism and automorphism structure of direct squares of

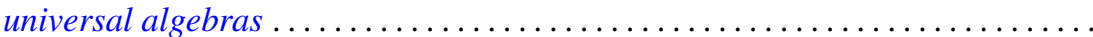

R. E. Harrell and Les Andrew Karlovitz, On tree structures in Banach spaces .....

Julien O. Hennefeld, Finding a maximal subalgebra on which the two Arens

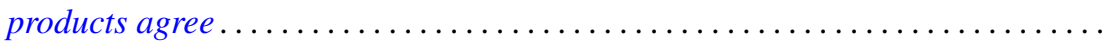

William Francis Keigher, Adjunctions and comonads in differential algebra .......

Robert Bernard Kelman, A Dirichlet-Jordan theorem for dual trigonometric

series

Allan Morton Krall, Stieltjes differential-boundary operators. III. Multivalued operators-linear relations...

Hui-Hsiung Kuo, On Gross differentiation on Banach spaces .

Tom Louton, A theorem on simultaneous observability ...

Kenneth Mandelberg, Amitsur cohomology for certain extensions of rings of algebraic integers.

Coy Lewis May, Automorphisms of compact Klein surfaces with boundary . . .

Peter A. McCoy, Generalized axisymmetric elliptic functions .

211

Muril Lynn Robertson, Concerning Siu's method for solving $y^{\prime}(t)=F(t$, $y(g(t)))$. .

Richard Lewis Roth, On restricting irreducible characters to normal

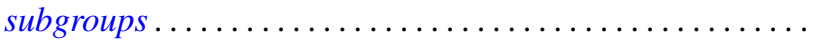

Albert Oscar Shar, $P$-primary decomposition of maps into an $H$-space .

Kenneth Barry Stolarsky, The sum of the distances to certain pointsets on the unit circle.

Bert Alan Taylor, Components of zero sets of analytic functions in $C^{2}$ in the unit ball or polydisc

Michel Valadier, Convex integrands on Souslin locally convex spaces ...

Januario Varela, Fields of automorphisms and derivations of $C$

Arnold Lewis Villone, A class of symmetric differential operators with deficiency

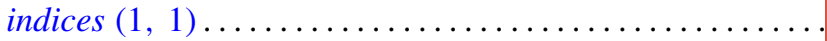

